\title{
Über das Vorkommen von Nahrungsalbumosen im Blut und im Urin.
}

\author{
Von
}

L. Borchardt.

Aus dem Institut für med. Chemie und exper. Pharmakologie zu Königsberg. Direktor: Ghr. Prof. Dr. Jaffe.)

(Der Redaktion zugegangen am 26. August 1908.)

Während wir durch zahlreiche Untersuchungen der letzten Jahre einen guten Einblick in die Verdauungsvorgänge innerhalb des Intestinaltraktus erhalten haben, sind unsere Kenntnisse über das Schicksal der Eiweißkörper jenseits der Darmwand noch immer sehr gering. Insbesondere ist die Frage, ob ein gewisser Teil des Nahrungseiweißes unverändert oder wenig verändert in Form von Albumosen die Darmwand passiert, bis in die jüngste Zeit eifrig diskutiert und noch nicht mit Sicherheit entschieden worden.

Die zahlreichen Versuche, Albumosen auf der Höhe der Verdauung im Blute nachzuweisen, haben - seien sie nun im positiven oder negativen Sinne ausgefallen - keine allgemeine Anerkennung erlangen können. Und es ist zweifellos, da $ß$ keine der angewendeten Methoden die Forderung, die zum sicheren Nachweis von Albumosen gemacht werden muß, voll und ganz erfültt: das Bluteiweiß vollständig und unverändert zu entfernen, ohne daß gleichzeitig auch Albumosen mit niedergeschlagen werden.

Ich habe deshalb versucht, diese Frage auf einem andern Wege zu lösen. Einer Idee Kossels folgend, habe ich ${ }^{1}$ ) das Schicksal einer wohlcharakterisierten Albumose, des Hemielastins, nach Aufnahme per os im Tierkörper verfolgt, und es ist mir gelungen, diese Albumose im Blut und in einigen Organen wiederzufinden.

1) Diese Zeitschrift, Bd. LI, S. 506, 1907.

Hoppe-Seyler's Zeitschrift f. physiol. Chemie. LVII. 
War somit die Frage nach dem Vorkommen von Albumosen im Blut zunächst prinzipiell in dem Sinne entschieden, daß Nahrungsalbumosen in die Blutbahn übergehen können (vorausgesetzt, daß das Hemielastin in dieser Hinsicht keine Sonderstellung einnimmt), so war es anderseits aus mancherlei Gründen wünschenswert,, diese Versuche zu erweitern und die Versuchsbedingungen den physiologischen Vorgängen noch mehr anzupassen. Besonders aber war mir maßgebend für die W'iederaufnahme dieser Versuche die Kritik, die meine Arbeit durch Abderhalden ${ }^{1}$ ) erfahren hatte. Abderhalden schreibt darüber: «Dieser Forscher hat die schöne Beobachtung gemacht, daß ein peptisches Abbauprodukt aus Elastin, das durch besondere Eigenschaften ausgezeichnet und daher in sehr geringen Mengen nachweisbar ist, sich im Blute bei seiner Einführung per os nachweisen läßt. Es liegt kein Grund vor, diesen Befund $\mathrm{zu}$ verallgemeinern. Borchardt hat nur nachgewiesen, daß das von ihm Hemielastin genannte Produkt in der Blutbahn und auch in Organen nachweisbar ist. Über die $\mathrm{Zu}-$ sammensetzung des Hemielastins wissen wir nichts. Es ist möglich, daß es überhaupt schwer angreif bar ist und deshalb unzerlegt im Organismus zirkuliert, $d$. h. es ist die Möglichkeit nicht ausgeschlossen, daß Hemielastin als Fremdkörper wirkt.» «Sollen diese Versuche für die «Albumosenfrage * von Bedeutung sein, d. h. für das normale Vorkommen von Albumosen im Blute grundlegend werden, dann wäre doch erst zu beweisen, $\mathrm{da} ß$ normalerweise Hemielastin im Blute vorkommt, wenn Elastin als solches verfüttert wird und nicht künstlich bereitetes Hemielastin.»

Diesen Nachweis habe ich jetzt erbracht. Auch nach Verfütterung von Elastin tritt Hemielastin regelmäßig in das Blut und die Organe über und ist mit der Hemielastinreaktion nachweisbar.

Die Versuchsanordnung entsprach durchaus den früher mitgeteilten Versuchen und ich kann hinsichtlich aller Details auf meine frühere Arbeit verweisen. Das zur Fütterung be-

1) Biochem. Zeitschrift, Bd. VIII, S. 369, 1908. 
nutzte Elastin wurde im Heidelberger physiol. Institut genau in der gleichen Weise dargestellt, wie das Elastin, das ich früher als Ausgangsmaterial für die Darstellung des Elastinverdauungsproduktes verwendete. Es gab keine Hemielastinreaktion.

Ich bin Herrn Prof. Kossel für die Überlassung des Materials zu besonderem Danke verpflichtet.

Das fast unlösliche Elastinpulver wurde in Milch suspendiert per Schlundsonde gegeben. Erbrechen trat niemals danach ein, ebensowenig Durchfall. In den ersten beiden Versuchen, in denen relativ sehr große Elastinmengen eingeführt wurden, lagen die Tiere danach träge und apathisch da und zeigten alle Zeichen hochgradiger Sättigung.

\section{Versuch.}

Ein $10^{1 / 2}$ Wochen altes weibliches Hündchen von $4450 \mathrm{~g}$ Körpergewicht erhält 26. 6. $0850 \mathrm{~g}$ Elastin per Schlundsonde. Wird nach 5 Stunden in Äthernarkose durch Entbluten aus der Pfortader getötet. $25 \mathrm{ccm}$ des Pfortaderbluts werden durch kurzes Aufkochen in schwach essigsaurer Lösung enteiweißt. Im Filtrat ist nach Einengen auf $15 \mathrm{ccm}$ die Hemielastinreaktion positiv. Auch in den Nieren, Milz, Leber, Magenwand, Dünndarm- und Dickdarmwand ist Hemielastin durch die charakteristische Reaktion wiederzufinden. Biuret- und Millonsche Probe sind positiv, die Glyoxylsäurereaktion negativ, nur in Magenund Darmwand positiv. Magen und Darm sind noch voll von unverdautem Elastin.

Durch diesen Versuch ist das Auftreten von Hemielastin im Blut und den Organen nach Fütterung mit Elastin beim jungen, noch nicht ausgewachsenen Tier erwiesen.

Das Vorkommen von Hemielastin in den Organen nach Elastinfütterung konnte nicht anders erklärt werden, als daß die Albumose nicht in der Leber festgehalten oder abgebaut, sondern in den großen Kreislauf gelangt war. Unter diesen Umständen mußte das Hemielastin auch im peripheren Blut vorhanden sein, wenn auch in größerer Verdünnung. Der erste in dieser Hinsicht unternommene Versuch an einem erwachsenen 
Tier ergab im Gegensatz zu allen späteren Versuchen ein völlig negatives Resultat.

\section{Versuch.}

Hündin von $5650 \mathrm{~g}$ Körpergewicht erhält am 30. 6. 08. $60 \mathrm{~g}$ Elastin in Milch per Schlundsonde. Nach 6 Stunden werden aus der Carotis $80 \mathrm{ccm}$ Blut entnommen, das in der üblichen Weise nach dem Enteiweißen auf dem Wasserbade eingeengt wird. Auch nach dem Einengen bis auf wenige Kubikzentimeter fällt weder die Hemielastinreaktion noch die Biuretreaktion positiv aus.

Der negative Ausfall dieses Versuches veranlaßte mich, dasselbe Tier nochmals mit Elastin zu füttern und das Hemielastin im Pfortaderblut und den Organen zu suchen. Hier war Hemielastin unter den gleichen Versuchsbedingungen leicht nachweisbar.

\section{Versuch.}

Die Hündin von Versuch 2 erhält 7. 7. 08 abermals $60 \mathrm{~g}$ Elastin in der gleichen Weise. Wird nach $6 \frac{1}{2}$ Stunden aus der Pfortader entblutet. $150 \mathrm{ccm}$ Pfortaderblut werden nach der üblichen Verdünnung mit der 10 fachen Menge Wasser enteiweißt; das Filtrat gibt nach Einengen auf $240 \mathrm{ccm}$ deutliche Hemielastinreaktion. Biuret- und Mill on sche Probe sind positiv, die Glyoxylsäurereaktion negativ. Nach weiterem Einengen des Filtrats auf $10 \mathrm{ccm}$ wird das Hemielastin durch Sättigen mit $\mathrm{NaCl}$ ausgesalzen. Es tritt ein deutlicher Niederschlag auf, von dem am anderen Tage abfiltriert wird. Der mit gesättigter Kochsalzlösung gewaschene Niederschlag wird in Wasser gelöst und gibt die Hemielastinreaktion sowie die Biuret- und Millonsche Probe.

Außer im Pfortaderblut war Hemielastin durch Hemielastin-, Biuret- und Millonsche Reaktion nachweisbar in Leber, Milz, Nieren, Pankreas, Magen- und Darmwand. Einige geschwollene Bauchlymphdrüsen, die in gleicher Weise behandelt wurden, gạben besonders deutliche Hemielastinreaktion.

Durch diesen Versuch war es erwiesen, daß auch beim erwachsenen Tier nach Elastinfütterung Hemielastin ins Blut 
und die Organe übertreten kann. Der negative Aưsfáll dès vorhergehenden Versuches an demselben Tier und unter dehselben Bedingungen ließ es aber wünschenswert erscheinen, durch Wiederholung einen Arhaltspunkt zú gewinnedn, ob der Übertritt der Albumose ins Blut zu den regelmäß̉igen Ërśchei= nungen gehört oder nur ausnahmsweise erfolgt. Zugleich wurae die Frage entschieden, daß auch im Carotisblut Hemielastin auf der Höhe der Elastinverdauung nachweisbär ist. Schließlich wurde in dem folgenden Versuch die Menge des verabreichten Elastins bedeutend herabgesetzt, um dem Einwand zu begegnen, daß nur nach Verabreichung von Elastinmengen, die das physiologische Maß überschreiten, Hemielastin in das Blut übertritt.

\section{Versuch.}

Männlicher Hund von $12910 \mathrm{~g}$ Körpergewicht erhält 14: 7.08 $50 \mathrm{~g}$ Elastin in Milch per Schlundsonde: Nach 6 Stunden werden aus der Carotis $210 \mathrm{ccm}$, gleich darauf aus der Pfortader $150 \mathrm{ccm}$ Blut entnommen. Im Pfortaderblut ist die Hemielastin= reaktion nach dem Enteiweißen noch vor dem Einengen (in $880 \mathrm{ccm}$ Flüssigkeit) deutlich, ebenso die Biuret- und Millonsche Reaktion, während die Glyoxylsäurereaktion auch nach dem Einengen auf $50 \mathrm{ccm}$ negativ ausfällt. Im Carotisblut isit nach Einengen auf $170 \mathrm{ccm}$ die Hemielastinreaktion positiv: Von Organen wurden Nieren, Milz, Pankreäs, Leber, Herz; Lungen, Muskeln auf Hemielastin untersucht; in allen diesen Organen konnte es nachgewiesen werden. Die Blase enthielt 200 ccm Urin. Derselbe gab weder die Hemielastinreaktion noch die Biuretprobe. Nach Einengen des Urins auf $100 \mathrm{ccm}$ war die Hemielastin- sowie die Biuretreaktion sehr deutlich.

Das Vorkommen von Hemielastin im Urin nach Elastinfütterung wurde durch den nächsten Versuch bestätigt.

5. Versuch.

Männlicher Terrier von $10750 \mathrm{~g}$ Körpergewicht erhält 31. 7. $0840 \mathrm{~g}$ Elastin in Milch per Schlundsonde. Nach 6 Stunden werden aus der Carotis $100 \mathrm{ccm}$ Blut entnommen, in welchem 
nach Einengen auf $80 \mathrm{ccm}$ die Hemielastin- sowie die Biuretreaktion positiv ausfallen.

Während des Aderlasses läßt der Hund Urin, von dem $100 \mathrm{ccm}$ aufgefangen werden. Der Urin ist eiweißfrei und gibt weder Hemielastin- noch Biuretreaktion. Nach dem Einengen auf $50 \mathrm{ccm}$ ist Hemielastin durch beide Reaktionen nachweisbar.

Das Resultat dieses Versuches wurde durch den folgenden Versuch bestätigt.

\section{Versuch.}

Derselbe Hund erhält 10. 8. 08 abermals $40 \mathrm{~g}$ Elastin. Nach 6 Stunden werden aus der Carotis $100 \mathrm{ccm}$ Blut entnommen, in dem nach Einengen auf $20 \mathrm{ccm}$ das Hemielastin durch die Hemielastin- sowie Biuretreaktion nachweisbar ist. Während des Aderlasses konnten wieder $80 \mathrm{ccm}$ Urin aufgefangen werden, die direkt sowohl Biuret- wie Hemielastinreaktion geben. Hemielastin- und Biuretreaktion sind auch in dem am anderen Morgen (ca. 20 Stunden nach der Elastinfütterung) entleerten Urin deutlich positiv. Der nächste Urin (32 Stunden nach der Elastinfütterung) enthielt noch nachweisbare Spuren von Hemielastin. Von da ab war der Urin frei davon.

Ich entnehme meinen Versuchen die folgenden Resultate: Sowohl beim jungen wie beim ausgewachsenen Tier tritt nach Fütterung mit nicht übermäßigen Mengen eines Eiweißkörpers, des Elastins, ein primäres Verdaungsprodukt desselben, das Hemielastin (Horbaczewski) oder Protoelastose (Chittenden und Hart) in sehr geringer Menge ins Pfortaderblut über. Von da gelangt es in den großen Kreislauf und zu den Körperorganen und kann mit dem Urin ausgeschieden werden.

Diese neuen und in mancher Hinsicht den herrschenden Anschauungen widersprechenden Resultate gestatten zunächst eine Beantwortung der von Abderhalden ${ }^{1}$ ) aufgeworfenen Frage: «Sollte der tierische Organismus nun auch normalerweise solche Produkte (gemeint ist das Hemielastin) in die Zirkulation bringen, um sie dann schließlich zum Teil durch

i) 1. c. 
die Nieren zu verlieren und zum Teil wieder zum Dünndarm zurückzuführen?» - Ich habe durch diese Versuche erwiesen, daß das Hemielastin z. T. durch die Nieren dem Körper verloren geht. Von dem Elastin der Nahrung gelangt ein kleiner Anteil ins Blut und wird durch die Nieren mit dem Urin ausgeschieden. $\mathrm{Ob}$ vollständig, $\mathrm{ob}$ ein Teil des ins Blut übergetretenen Hemielastins in den Organen abgebaut oder zu diesem Zwecke zur Darmwand zurückgeführt wird, entzieht sich einstweilen noch unserer Kenntnis.

Wichtiger ist natürlich für die Bedeutung dieser Versuche der Einwand, daß das Hemielastin möglicherweise als Fremdkörper wirkt und sich anders verhält wie andere Albumosen. Die Möglichkeit einer solchen Sonderstellung des Hemielastins unter den Albumosen erscheint mir aber schon aus theoretischen Gründen ganz ausgeschlossen. Es ist nicht einzusehen, warum die Elastinverdauung bereits bei dem ersten Verdauungsprodukt Halt machen sollte, weshalb der Körper dieses dann eliminieren müßte, weil er es nicht weiter abbauen kann. Zudem ist es durch künstliche Verdauungsversuche von Morochowetz, ${ }^{1}$ ) Richards und Gies ${ }^{2}$ ) erwiesen, daß tatsächlich die Elastinverdauung weiter geht als bis zum Hemielastin. Wenn das aber nicht der Fall wäre, so wäre es doch näherliegend, anzunehmen, daß das aus der Nahrung entstandene Hemielastin mit dem Stuhl ausgeschieden werde und nicht in die Blutbahn überginge. Der Übergang der Albumose ins Blut wäre also auch dann ein bemerkenswertes Novum. Schließlich muß ich aber der Angabe Abderhaldens widersprechen, daß wir über die Zusammensetzung des Hemielastins nichts wüßten. Zwar liegt eine quantitative Bestimmung der Bausteine des Hemielastins bisher nicht vor; die Albumosennatur und die chemischen Eigenschaften des Hemielastins sind aber durch Horbaczewski, ${ }^{3}$ ) Ghittenden und Hart ${ }^{ \pm}$) eingehend studiert.

1) St. Petersb. med. Wochenschr. 1886. - Ref. n. Malys Jahresber., Bd. XVI, S. 271. 1886.

2) Amer. Journ. of physiol., Bd. VII, S. 93. 1902.

3) Diese Zeitschrift, B. VI, S. 330. 1882.

4) Zeitschrift f. Biol., Bd. XXV, S. 368. 1889. 
312 L. Borchardt, Über Nahrungsalbumosen im Blut und im Urin.

Einer Verallgemeinerung meiner Versuche, d. h. der Annahme; daß nach Eiweißfütterung geringe Mengen der Nahrungsalbumosen ins Blut übergehen, steht nun aber - wie ich meine - ein Bedenken entgegen.

Es könnten qualitative Verschiedenheiten unter den der Nahrung entstammenden Albumosen bestehen; es wäre denkbar, daß gewisse Albumosen leichter, andere schwerer oder garnicht die Darmwand passieren und ins Blut gelangen, und eine solche Annahme fände eine Parallele in der verschieden guten Durchlässigkeit der Darmwand für die einzelnen Zuckerarten. Über Versuche, die ich in diesem Sinne unternommen habe, will ich später berichten.

In dieser Arbeit begnüge ich mich damit, festzustellen, daß nach Einführung eines Eiweißkörpers, des Elastins, das auch in der gewöhnlichen Nahrung eine nicht unwesentliche Rolle spielt, per os in nicht abundanter Menge eine Albumose, Hemielastin, ins Blut übergeht, welche schließlich durch die Nieren mit dem Urin ausgeschieden wird. 